\title{
Case Report: Globus Pallidus Internus (GPi) Deep Brain Stimulation Induced Keyboard Typing Dysfunction
}

\author{
Joshua K. Wong*, Melissa J. Armstrong, Leonardo Almeida, Aparna Wagle Shukla, \\ Addie Patterson, Michael S. Okun and Irene A. Malaty
}

Department of Neurology, McKnight Brain Institute, Norman Fixel Institute for Neurological Diseases, University of Florida, Gainesville, FL, United States

OPEN ACCESS

Edited by:

lone Fine,

University of Washington,

United States

Reviewed by:

Chencheng Zhang,

Shanghai Jiao Tong University, China

Thomas M. Kinfe,

University of Erlangen Nuremberg,

Germany

*Correspondence: Joshua K. Wong joshua.wong@neurology.ufl.edu

Specialty section: This article was submitted to Brain Imaging and Stimulation, a section of the journal Frontiers in Human Neuroscience

Received: 14 July 2020 Accepted: 12 October 2020 Published: 09 November 2020

Citation: Wong JK, Armstrong MJ, Almeida L, Wagle Shukla A, Patterson A, Okun MS and Malaty IA (2020) Case Report: Globus Pallidus Internus (GPi)

Deep Brain Stimulation Induced Keyboard Typing Dysfunction. Front. Hum. Neurosci. 14:583441. doi: 10.3389/fnhum.2020.583441
Introduction: Typing on a keyboard requires complex collaboration between visuospatial/procedural memory, language, and motor function. The impaired ability to type, independent of motor deficits, apraxia, or aphasia has been coined "dystypia."

Case Presentation: A 68-year-old woman with a history of blepharospasm, oromandibular, and segmental dystonia underwent bilateral pallidal deep brain stimulation (DBS) because of a waning response to botulinum toxin therapy. Following DBS, she discovered she no longer "remembered" how to type fluidly and had to "hunt and peck" for letters on the keyboard. This issue persisted at a 2-year follow-up. The patient underwent serial typing tests with the DBS ON vs. OFF. Post-operative lead reconstruction was performed using Lead-DBS. The volume of tissue activation (VTA) modeling was combined with whole-brain tractography.

Results: Typing improved when the device was switched to the DBS OFF state. Cortical mapping revealed strong modulation of the right angular gyrus, left calcarine fissure, and left cuneus. There was also activation of bilateral supplemental motor areas and superior parietal gyri.

Discussion: Shared lesion topography analysis of dystypia cases in the literature has suggested the involvement of the superior longitudinal fasciculus (SLF). The SLF involves the superior parietal lobe, angular gyrus, supramarginal gyrus, and arcuate fasciculus. Our patient's connectivity pattern suggested SLF involvement. The improvement in OFF state typing and her imaging together suggested that the dystypia in her case was a stimulation-induced side effect.

Conclusion: Dystypia is a rare side effect of Globus Pallidus Internus (GPi) DBS therapy and may be associated with SLF involvement.

Keywords: deep brain stimulation, dystonia, dystypia, connectomics, stimulation induced side effect 


\section{INTRODUCTION}

Typing on a keyboard is a complex coordinated function that requires network coordination between visuospatial procedural memory, language, and motor function (Ryu et al., 2012; Cook et al., 2013). The impaired ability to type, when manifested independently of motor deficits, apraxia, or aphasia, has been coined "dystypia" (Otsuki et al., 2002). Dystypia can be disruptive and impact activities of daily living especially given the heavy use of technology. In light of recent reports of swimming dysfunction following deep brain stimulation (DBS), we report a case of dystypia (keyboard typing dysfunction) as a potential side effect of DBS therapy (Waldvogel et al., 2020).

\section{CASE PRESENTATION}

A 68-year-old woman with a history of blepharospasm, oromandibular and segmental dystonia underwent bilateral pallidal DBS. Before DBS she had a waning response to botulinum toxin therapy. In the acute stage of her recovery from the DBS surgery, she reported "forgetting how to type on the computer." At baseline, she was proficient in typing as she did not have dystonia symptoms in the arm. Her previous occupation was administrative office work which involved copious typing. Within weeks following DBS implantation, she reported she no longer "remembered" how to type fluidly and had to "hunt and peck" for letters on the keyboard. This issue persisted into the 2-years of follow-up we have for her post-DBS. She did not have issues with any other motor tasks.

The clinicians performed the following testing to sort out the dysfunction and the relationship to DBS activation or inactivation (three conditions): (1) DBS ON; (2) 10 min following the DBS OFF condition; and (3) $20 \mathrm{~min}$ following the DBS OFF condition. Informed consent for testing and videotaping was obtained from the patient. Visuospatial testing (e.g., Trail Making B test, cube copy, clock drawing) and the neurological exam were performed in the DBS ON state and it was confirmed that there was no evidence of visuospatial neglect or aphasia. She performed three different 1-min online typing tests which were provided by the National Computer Science Academy (NCSA, Whitesboro, TX, USA). The tests can be viewed in the Supplementary Video 1. The three segments show DBS ON, 10-min washout, and 20-min washout. The testing duration was limited by the return of the patient's pre-operative dystonia symptoms.

A post-operative DBS lead location analysis was performed using the Lead-DBS advanced image processing pipeline (Horn and Kühn, 2015; Horn et al., 2019) ${ }^{1}$. The volume of tissue activation (VTA) was modeled and estimated based on the patient's most recent DBS programming parameters [Left Globus Pallidus Internus (GPi): $1-\mathrm{C}+3.5 \mathrm{~V}, 150 \mu \mathrm{s}, 120 \mathrm{~Hz}$; Right GPi: $9-\mathrm{C}+2.5 \mathrm{~V}, 150 \mu \mathrm{s}, 120 \mathrm{~Hz}$ ]. Whole-brain deterministic tractography was computed in DSI Studio using a diffusion MRI population-averaged template from the Human Connectome

\footnotetext{
${ }^{1}$ https://www.lead-dbs.org/
}

Project (HCP-1021; Yeh et al., 2013, 2018). The VTA was used as a seed for fiber tracking and resulting activated fiber tracks were calculated.

\section{RESULTS}

Video testing of the patient's typing was performed at 2 years and 7 months post-implantation. The DBS programming settings at the time of testing were unchanged for the previous 2 years. This setting was reported to provide maximal benefit for her dystonia symptoms. Based on the latest settings, she reported complete or near-complete resolution of her dystonia symptoms except for her mild dystonic tremor in the right arm.

As the washout time increased (DBS OFF), she reported increased confidence in her typing abilities and less dependence on looking down at the keyboard. Her typing speed also increased from 0.6 words per minute (WPM) at baseline to 2.6 WPM and 3.8 WPM after turning the DBS OFF for 10 and $20 \mathrm{~min}$, respectively. She felt typing improved OFF DBS but not back to her pre-DBS baseline.

The fiber tract activation pattern from the patient's DBS lead location analysis is shown in Figure 1. Cortical parcellation mapping revealed a strong modulation of the right angular gyrus, left calcarine fissure, and left cuneus. There was the activation of bilateral supplemental motor areas and the superior parietal gyri.

\section{DISCUSSION}

Several case reports have described "dystypia" in the absence of motor weakness, apraxia, and aphasia (Otsuki et al., 2002; Ryu et al., 2012; Cook et al., 2013; Thomas and Mestre, 2017; Sharma et al., 2019). Typically, dystypia has been reported in the setting of acute stroke. One case report described a patient with dystypia following bilateral subthalamic nucleus (STN) DBS (Lee et al., 2016). Post-operative imaging revealed prominent vasogenic edema in the frontal lobe surrounding the left STN DBS lead. In contrast to this case, Lee et al. (2016) attributed the dystypia to frontoparietal network dysfunction secondary to peri-electrode edema rather than a stimulationinduced side effect of STN DBS. The authors observed a marked improvement in dystypia following resolution of the peri-electrode edema. A recent literature review investigating the shared lesion topography of dystypia cases proposed involvement of the superior longitudinal fasciculus (SLF; Sharma et al., 2019). The SLF has been characterized to involve the superior frontal lobe, dorsal prefrontal region, superior parietal lobe, the angular gyrus, the supramarginal gyrus, and the arcuate fasciculus (Makris et al., 2005). A comparison to our patient's connectivity pattern also revealed the involvement of the SLF as a possible explanation for her dystypia.

We acknowledge several limitations of this study. First, we recognize our case is an $\mathrm{N}$-of- 1 observation and that these results should be interpreted with caution. However, the findings in our study highlight that there may be underrecognized stimulationinduced DBS related side effects. As these impairments appear to be task-specific and patient-specific, we appreciate the need for a larger, more robust exploration into stimulation-induced 


\section{Cortical activation profile of pallidal stimulation induced dystypia}

A

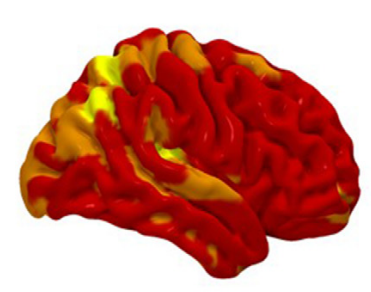

Right lateral view
B

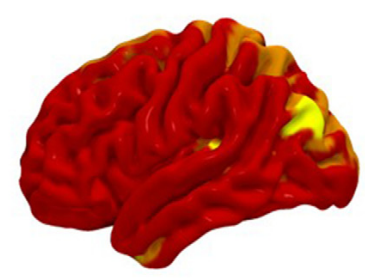

Left lateral view
C

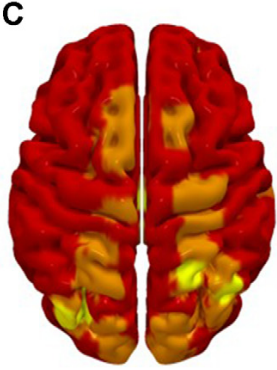

Superior view

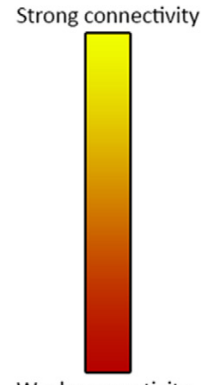

Weak connectivity

FIGURE 1 | The cortical activation pattern of bilateral globus pallidus internus (GPi) deep brain stimulation (DBS) is demonstrated. The (A) right lateral view (B) left lateral view and $\mathbf{( C )}$ superior view are shown. The activation pattern is based on the patient's DBS device programming parameters. There was a strong activation of the right angular gyrus, left calcarine fissure, and left cuneus and moderate activation of bilateral supplemental motor areas and the superior parietal gyri.

side effects. Second, due to limitations in the MRI acquisition, our connectomic analysis utilized population-averaged template data rather than patient-specific data. Future studies should incorporate both approaches to see if there are any differences in connectivity results. Finally, we acknowledge the need in future cases for a comprehensive neuropsychological testing battery to further characterize dystypia.

Our findings suggest that dystypia is a rare stimulationinduced side effect of GPi DBS therapy and may be related to SLF involvement. This phenomenon appears different than GPi induced parkinsonism, however, comparative studies should be performed to observe if parkinsonism involves a common network pathway (Blahak et al., 2011; Schrader et al., 2011). Although the fundamental phenomenology between dystypia and parkinsonism is different, the examination of the active contacts, lead location, and fiber bundles stimulated by GPi DBS may reveal overlapping connectivity profiles or modulation of interconnected nodes along the same network. Recent reports of DBS-induced swimming dysfunction may share common underpinnings and this approach may be useful to better understand this phenomenon (Waldvogel et al., 2020). Finally, since the majority of centers have yet to formally assess for dystypia post-DBS, the prevalence of this DBS side effect remains unknown.

\section{DATA AVAILABILITY STATEMENT}

The datasets presented in this article are not readily available. Requests to access the datasets should be directed to joshua.wong@neurology.ufl.edu.

\section{ETHICS STATEMENT}

Ethical review and approval was not required for the study on human participants in accordance with the local legislation and institutional requirements. The patients/participants provided their written informed consent to participate in this study.

\section{AUTHOR CONTRIBUTIONS}

JW designed the study, acquired and analyzed the data, and drafted the manuscript. MA, LA, AW, AP, and MO revised the manuscript. IM designed the study and revised the manuscript. All authors contributed to the article and approved the submitted version.

\section{FUNDING}

This research did not receive any specific grant from funding agencies in the public, commercial, or not-for-profit sectors. JW's DBS research is supported by R25 NS108939. MA reports receipt of personal fees from the American Academy of Neurology (consultancy services); grants from the Lewy Body Dementia Association; the Michael J. Fox Foundation; funding from the Agency of Healthcare Research and Quality; and others from Oxford University Press (royalties) outside the submitted work. LA serves as a consultant and has received honoraria from Medtronic and Boston Scientific. AW reports grants from the NIH and has received grant support from Benign Essential Blepharospasm Research Foundation, Dystonia Coalition, Dystonia Medical Research Foundation, National Organization for Rare Disorders, and grant support from NIH (KL2 and K23 NS092957-01A1). Reports receiving honoraria from Acadia, Cavion, Elsevier, and MJFF in past; participates as a co-investigator for several NIH, foundations, and industry-sponsored trials over the years but has not received honoraria. MO serves as a consultant for the Parkinson's Foundation and has received research grants from NIH, Parkinson's Foundation, the Michael J. Fox Foundation, the Parkinson Alliance, Smallwood Foundation, the Bachmann-Strauss Foundation, the Tourette Syndrome Association, and the UF Foundation. MO's DBS research is supported by R01 NR014852 and R01NS096008. MO has received royalties for publications with Demos, Manson, Amazon, Smashwords, Books4Patients, Perseus, Robert Rose, 
Oxford, and Cambridge (movement disorders books). MO is an associate editor for the New England Journal of Medicine Journal Watch Neurology. MO has participated in CME and educational activities on movement disorders sponsored by the Academy for Healthcare Learning, PeerView, Prime, QuantiaMD, WebMD/Medscape, Medicus, MedNet, Einstein, MedNet, Henry Stewart, American Academy of Neurology, Movement Disorders Society and by Vanderbilt University. The institution and not MO receives grants from Medtronic, Abbvie, Abbott, and Allergan and the principal investigator (PI) has no financial interest in these grants. MO has participated as a site PI and/or co-investigator for several NIH, foundations, and industry-sponsored trials over the years but has not received honoraria.

\section{REFERENCES}

Blahak, C., Capelle, H.-H., Baezner, H., Kinfe, T. M., Hennerici, M. G., and Krauss, J. K. (2011). Micrographia induced by pallidal DBS for segmental dystonia: a subtle sign of hypokinesia? J. Neural Transm. 118, 549-553. doi: 10.1007/s00702-010-0544-y

Cook, F. A. B., Makin, S. D. J., Wardlaw, J., and Dennis, M. S. (2013). Dystypia in acute stroke not attributable to aphasia or neglect. BMJ Case Rep. 2013:bcr2013200257. doi: 10.1136/bcr-2013-200257

Horn, A., and Kühn, A. A. (2015). Lead-DBS: a toolbox for deep brain stimulation electrode localizations and visualizations. NeuroImage 107, 127-135. doi: 10.1016/j.neuroimage.2014.12.002

Horn, A., Li, N., Dembek, T. A., Kappel, A., Boulay, C., Ewert, S., et al. (2019). Lead-DBS v2: towards a comprehensive pipeline for deep brain stimulation imaging. NeuroImage 184, 293-316. doi: 10.1016/j.neuroimage.2018.08.068

Lee, S.-H., Jeon, K., Son, B., and Kim, J.-S. (2016). Isolated dystypia after subthalamic nucleus deep brain stimulation in a patient with Parkinson's disease. Acta Neurochir. 158, 783-784. doi: 10.1007/s00701-016-2716-5

Makris, N., Kennedy, D. N., McInerney, S., Sorensen, A. G., Wang, R., Caviness, V. S., et al. (2005). Segmentation of subcomponents within the superior longitudinal fascicle in humans: a quantitative, in vivo, DT-MRI study. Cereb. Cortex 15, 854-869. doi: 10.1093/cercor/bhh186

Otsuki, M., Soma, Y., Arihiro, S., Watanabe, Y., Moriwaki, H., and Naritomi, H. (2002). Dystypia: isolated typing impairment without aphasia, apraxia or visuospatial impairment. Eur. Neurol. 47, 136-140. doi: 10.1159/000047971

Ryu, D.-W., Kim, J.-S., Yang, D.-W., Kim, Y.-I., and Lee, K.-S. (2012). Dystypia without aphasia associated with visuospatial memory impairment in a patient with acute stroke. Alzheimer Dis. Assoc. Disord. 26, 285-288. doi: 10.1097/WAD.0b013e318231e614

Schrader, C., Capelle, H.-H., Kinfe, T. M., Blahak, C., Bäzner, H., Lütjens, G., et al. (2011). GPi-DBS may induce a hypokinetic gait disorder with freezing of gait in patients with dystonia. Neurology 77, 483-488. doi: 10.1212/WNL. ob013e318227b19e

\section{SUPPLEMENTARY MATERIAL}

The Supplementary Material for this article can be found online at: https://www.frontiersin.org/article/10.3389/fnhum. 2020.583441/full\#supplementary-material.

SUPPLEMENTARY VIDEO 1 | Pallidal deep brain stimulation (DBS)-induced dystypia. The patient in the video begins by taking a typing test with the DBS ON. Her hands are positioned off-center and she frequently looks down at the keyboard for visual confirmation of each keystroke before pressing down. She primarily uses her index finger to type and must pause to double-check her progress on the screen. After turning the DBS OFF for $10 \mathrm{~min}$, her fingers are positioned over the "home row" keys and she can type more fluidly. She does not constantly look down at the keyboard. After turning the DBS OFF for $20 \mathrm{~min}$, she incorporates all fingers into her typing and she spends most of the time viewing the screen.

Sharma, A. K., Fridman, S., Gleichgerrcht, E., and Sposato, L. A. (2019). Dystextia and dystypia as modern stroke symptoms: a case series and literature review. Clin. Neurol. Neurosurg. 180, 25-27. doi: 10.1016/j.clineuro.2019. 02.001

Thomas, N. W. D., and Mestre, T. A. (2017). Impact of new technologies in a stroke presentation: a case of dystextia and dystypia. Can. J. Neurol. Sci. 44, 458-460. doi: $10.1017 /$ cjn.2016.431

Waldvogel, D., Baumann-Vogel, H., Stieglitz, L., Hänggi-Schickli, R., and Baumann, C. R. (2020). Beware of deep water after subthalamic deep brain stimulation. Neurology 94, 39-41. doi: 10.1212/WNL.0000000000008664

Yeh, F.-C., Panesar, S., Fernandes, D., Meola, A., Yoshino, M., FernandezMiranda, J. C., et al. (2018). Population-averaged atlas of the macroscale human structural connectome and its network topology. NeuroImage 178, 57-68. doi: 10.1016/j.neuroimage.2018.05.027

Yeh, F.-C., Verstynen, T. D., Wang, Y., Fernández-Miranda, J. C., and Tseng, W.-Y. I. (2013). Deterministic diffusion fiber tracking improved by quantitative anisotropy. PLoS One 8:e80713. doi: 10.1371/journal.pone.00 80713

Conflict of Interest: The authors declare that the research was conducted in the absence of any commercial or financial relationships that could be construed as a potential conflict of interest.

The reviewer CZ declared a past co-authorship with several of the authors LA, MO to the handling Editor.

Copyright (c) 2020 Wong, Armstrong, Almeida, Wagle Shukla, Patterson, Okun and Malaty. This is an open-access article distributed under the terms of the Creative Commons Attribution License (CC BY). The use, distribution or reproduction in other forums is permitted, provided the original author(s) and the copyright owner(s) are credited and that the original publication in this journal is cited, in accordance with accepted academic practice. No use, distribution or reproduction is permitted which does not comply with these terms. 